\title{
Periorbital Necrotizing Fasciitis Caused by Streptococcus Viridians
}

\author{
Belinda Pustina, Naser Salihu \\ Department of Ophthalmology, University Clinical Center of Kosovo, Prishtina, Kosovo \\ Email: belindapustina@gmail.com
}

Received 22 April 2015; accepted 19 May 2015; published 22 May 2015

Copyright (C) 2015 by authors and Scientific Research Publishing Inc.

This work is licensed under the Creative Commons Attribution International License (CC BY). http://creativecommons.org/licenses/by/4.0/

(c) () Open Access

\begin{abstract}
Necrotizing fasciitis (NF) is a life threatening soft tissue infection characterized by necrosis of fascia and subcutaneous tissue. If this disease is misdiagnosed and mistreated, because of the fast spreading, it can lead to death. Prompt treatment with antibiotics and surgical debridement is necessary and lifesaving in this disease. In uncomplicated cases and early stages of the disease it can be treated only with intravenous antibiotics. This study presents a 70-year-old female, farmer who approached in our clinic with edema of periorbital region and a minor trauma in her lower eyelid. First skin anthrax of eyelids was considered in differential diagnosis, because the patient was in contact with animals. Culture taken from the wound resulted positive for Streptococcus viridians $\alpha$ hemolytic Streptococcus: the viridians group. Treatment with Ceftriaxone + Penicillin was initiated immediately. Patient responded to intravenous antibiotics and after 2 days the edema began to regress. After 2 weeks patient was discharged from the hospital and the clinical outcome was satisfactory. Based on this case early diagnosed necrotizing fasciitis may be treated only with antibiotics.
\end{abstract}

Keywords

Periorbital Necrotizing Fasciitis, Streptococcus Viridians, Minor Trauma

\section{Introduction}

Periorbital NF caused by Streptococcus viridians is a severe and uncommon bacterial infection primary involving superficial fascia and subcutaneous tissue. Minor trauma may be the way for bacteria to enter subcutaneous tissue.

Treatment of necrotizing fasciitis involves intravenous antibiotics and surgical debridement. Delayed treatment is associated with increased morbidity and mortality [1]. Streptococcus produces toxins in the blood which 
causes Systemic Inflammatory Response (SIRS) that may lead to septic shock [2] and death [3] [4].

Even though aggressive surgical debridement should be started as soon as possible, there are some case reports supporting the evidence that in selected uncomplicated cases there is no need for aggressive surgical debridement in necrotizing fasciitis. They can be treated only with antibiotics [5]-[7].

In this study we represent a case of periorbital NF after minor trauma that was successfully treated only with systemic antibiotics, because it was diagnosed in early stages.

\section{Case Presentation}

A 70-year-old female farmer with diabetes was presented in our clinic with edema of the eyelids and right periorbital region. She came to our clinic in a febrile and lethargic condition. Diffuse eyelid swelling was noticed. Although she had skin anesthesia she was complaining for the pain. The skin was pink to purple color. On clinical examination she had edema and discharge in her upper eyelid and lower eyelid. In the lower eyelid there was a minor wound that looked like an insect bite (Figure 1). It was ulcerated with the crust and had raised margins. Crusts and necrosis was noticed also (Figure 2). Since she was at contact with animals, and farmers are at great risk of exposure to infected materials of animals, skin anthrax was considered.

Culture was taken from the wound and resulted positive for Streptococcus Viridians. Intravenous Ceftriaxone $2 \mathrm{~g} \mathrm{q12} \mathrm{h}$ and Penicilin G 4 milion q4h units IV in three divided doses was applied immediately. The clinical signs regressed only with antibiotic therapy. After two days the edema started to regress and the clinical condition started to improve (Figure 3). Eyes were also examined and there were no pathological findings in her right eye. The visual acuity and intraocular tension were normal.

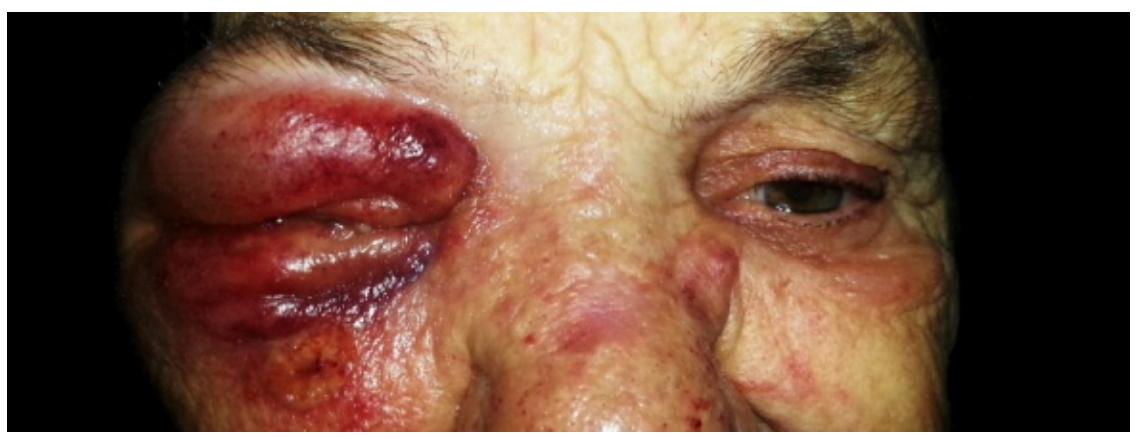

Figure 1. Periorbital edema with tissue necrosis, violet discoloration and fluid filled bullae.

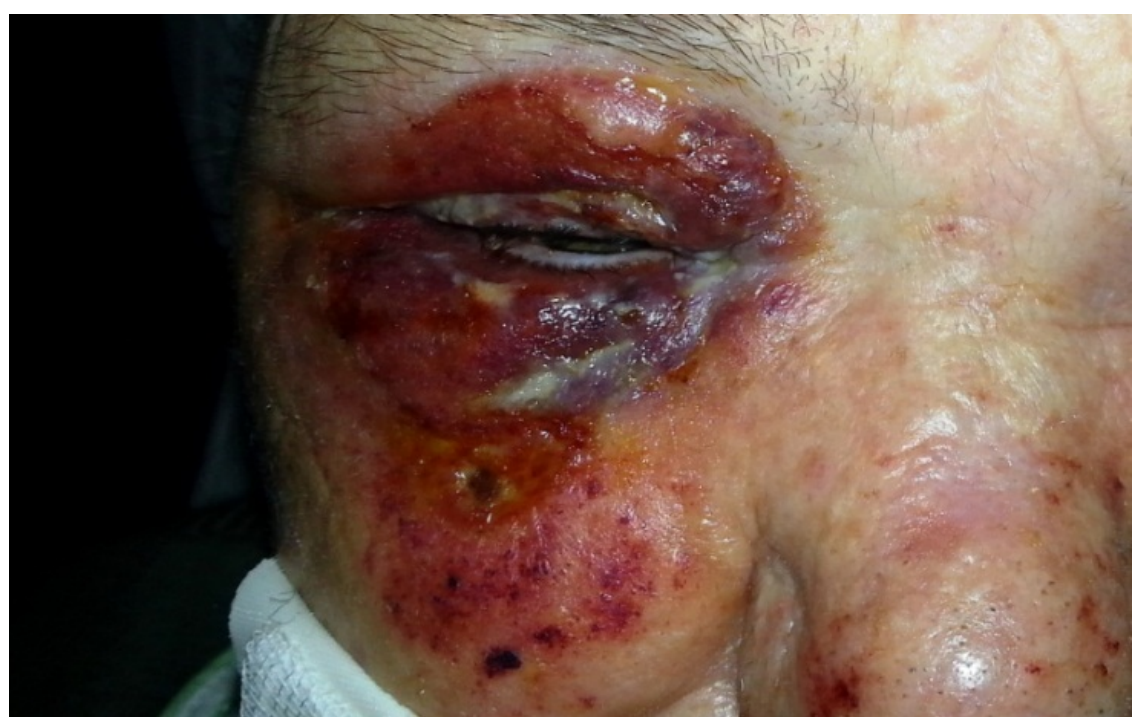

Figure 2. Crusts and necrosis of eyelids. 


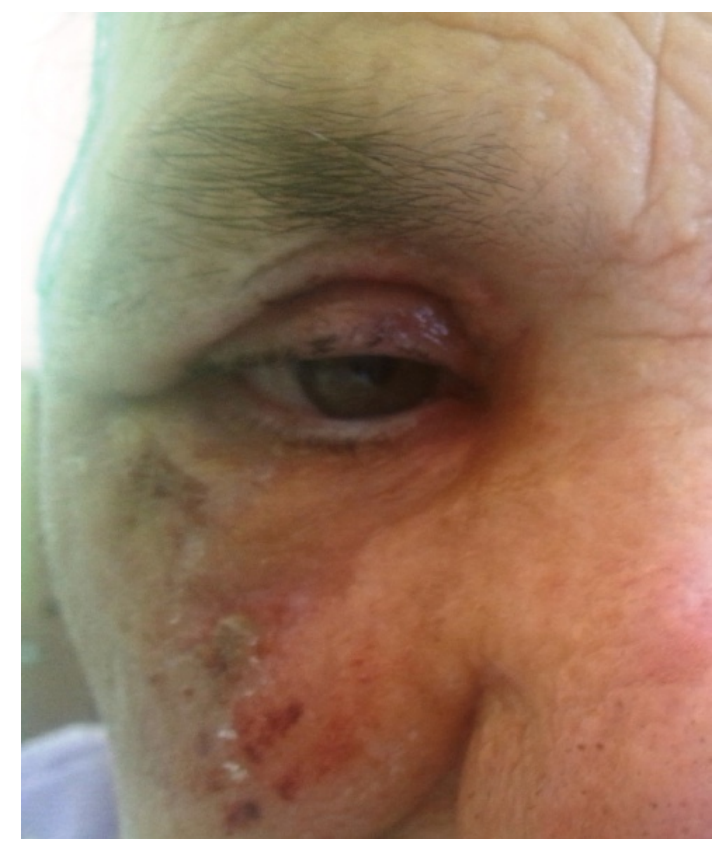

Figure 3. Regression of edema and necrosis of eyelids.

\section{Discussion}

Based on the involved layer infections of periorbital soft tissue with necrosis can be classified as cellulitis, fasciitis or myositis [8]. NF is a rare, rapidly progressive infection of soft tissue. Periorbital NF is uncommon because of the excellent blood supply to that area [9].

There are some types of the NF. Type I infections are polymicrobial, they are more often and tend to occur in patients with significant co-morbidities such as diabetes or cancer in which host defenses are compromised favoring the development of these infections.

Type II infections are caused by group A Streptococcus and are most common in otherwise healthy individuals with a history of trauma, intravenous drug abuse or surgery [10].

Rarity of the disease leads to misdiagnosis and mistreatment. Necrotizing fasciitis should not be misdiagnosed with preseptal cellulitis and orbital cellulitis. The differences between them are presence of cyanosis (violet discoloration) and serous fluid filled bullae [11].

Antibiotics may not be enough, because of the possibility of thrombosis in blood vessels in that region, that's why debridement is needed in complicated cases. To avoid ectropion and keratitis during debridement underlying muscles and eyelids must be protected [12] [13].

Based on a BOSU study in UK an incidence of 0.24 per 1.000.000 NF per annum was identified over a 2-year period. $\beta$ hemolytic Streptococcus A was identified in $76 \%$ and systemic complications in $66.66 \%$ with sepsis and death occurred in $10 \%$ [14].

It is reported mainly in adults with a female predominance $54 \%$ were about one half previously healthy. Following blunt trauma were reported $17 \%$, penetrating injury $22 \%$ and $11 \%$ after face surgery. In $28 \%$ no cause was identify [15].

Even though periorbital NF is a rare disease it should be considered after minor trauma in patients with weaken immune system as in this case with diabetes.

It is difficult to differentiate it from other common soft tissue infections. One of the infections that should be considered is skin anthrax. It is important to have suspicions especially in cases with history of contact with animals or animal products. Although the history is very important in putting the right diagnosis gram staining and culture confirm the diagnosis [16].

Periorbital redness and edema leading to a formation of large bullae and skin color progressing from rose to blue gray are characteristics of periorbital NF. Also one of the signs that can lead to diagnosis of NF is complaint of pain and anesthesia over the affected area and swelling around the lesion. One of the signs that can in- 
crease the suspicion of the skin anthrax is black eschar, not seen in NF [17] [18].

In conclusion periorbital NF is a rare disease and if it's not diagnosed in early stages it can cause serious and life threatening problems. The viridians group of $\alpha$ hemolytic Streptococcus should be considered in etiology of periorbital necrotizing fasciitis after minor trauma in patients with co-morbidities. Warning signs that can lead us to right diagnosis are violet skin discoloration and skin anesthesia. Early treatment may prevent functional and esthetic side effects.

\section{Conclusion}

Necrotizing fasciitis in early and uncomplicated stages may be treated successfully only with conservative treatment.

\section{Disclosure}

The authors declare that there is no conflict of interests regarding the publication of this paper.

\section{References}

[1] Lazzeri, D., Lazzeri, S., Figus, M., et al. (2010) Periorbital Necrotising Fasciitis. British Journal of Ophthalmology, 94, 1577-1585. http://dx.doi.org/10.1136/bjo.2009.167486

[2] Bustos, B.R., Soto, G.G., Hickmann, O.L. and Torres, B.C. (2009) Necrotizing Fasciitis of the Eyelids and Toxic Shock Syndrome Due to Streptococcus pyogenes. Revista Chilena de Infectología, 26, 152-155.

[3] Stevens, D.L. (2000) Streptococcal Toxic Shock Syndrome Associated with Necrotizing Fasciitis. Annual Review of Medicine, 51, 271-288. http://dx.doi.org/10.1146/annurev.med.51.1.271

[4] Sartelli, M. (2010) A Focus on Intra-Abdominal Infections. World Journal of Emergency Surgery, 5, 9. http://dx.doi.org/10.1186/1749-7922-5-9

[5] Majeski, J.A. and Alexander, J.W. (1983) Early Diagnosis, Nutritional Support and Immediate Extensive Debridement Improve Survival in Necrotizingfasciitis. American Journal of Surgery, 145, 784-787. http://dx.doi.org/10.1016/0002-9610(83)90140-X

[6] Lee Hooi, L., Hou, B.A. and Lay Leng, S. (2012) Group A Streptococcus Necrotizing Fasciitis of the Eyelid: A Case Report of Good Outcome with Medical Management. Ophthalmic Plastic Reconstructive Surgery, 28, e13-e15. http://dx.doi.org/10.1097/IOP.0b013e31821282ee

[7] Pessa, M.E. and Howard, R.J. (1985) Necrotizing Fasciitis. Surgery, Gynecology Obstetrics, 161, 357-361.

[8] McHenrv, C.R. and Malangoni, M.A. (1995) Necrotizing Soft Tissue Infections. In: Fry, D.E., Ed., Surgical Infections, Little, Brown and Co., Boston, 161-168.

[9] Kihiczak, G.G., Schwartz, R.A. and Kapila, R. (2006) Necrotizing Fasciitis: A Deadly Infection. Journal of the European Academy of Dermatology and Venereology, 20, 365-369. http://dx.doi.org/10.1111/j.1468-3083.2006.01487.x

[10] Kronish, J.W. and McLeish, W.M. (1991) Eyelid Necrosis and Periorbital Necrotizing Fasciitis. Report of a Case and Review of the Literature. Ophthalmology, 98, 92-98. http://dx.doi.org/10.1016/S0161-6420(91)32334-0

[11] Lazzeri, D., Lazzeri, S., Figus, M., et al. (2010) Periorbital Necrotizing Fasciitis. British Journal of Ophthalmology, 94, 1577-1585. http://dx.doi.org/10.1136/bjo.2009.167486

[12] Saldana, M., Gupta, D., Khandwala, M., Weir, R. and Beigi, B. (2010) Periorbital Necrotizing Fasciitis: Outcomes Using a CT-Guided Surgical Debridement Approach. European Journal of Ophthalmology, 20, 209-214.

[13] Stone, H.H. and Martin, J.D. (1972) Synergistic Necrotizing Cellulitis. Annals of Surgery, 175, 702-711. http://dx.doi.org/10.1097/00000658-197205000-00010

[14] Flavahan, P.W., Cauchi, P., Gregory, M.E., Foot, B. and Drummond, S.R. (2014) Incidence of Periorbital Necrotising Fasciitis in the UK Population: A BOSU Study. British Journal of Ophthalmology, 98, 1177-1180. http://dx.doi.org/10.1136/bjophthalmol-2013-304735

[15] Lazzeri, D., Lazzeri, S., Figus, M., Tascini, C., Bocci, G., Colizzi, L., Giannotti, G., Lorenzetti, F., Gandini, D., Danesi, R., Menichetti, F., Del Tacca, M., Nardi, M. and Pantaloni, M. (2010) Periorbital Necrotising Fasciitis. British Journal of Ophthalmology, 94, 1577-1585. http://dx.doi.org/10.1136/bjo.2009.167486

[16] Karahocagil, M.K., Akdeniz, N., Akdeniz, H., Calka, O., Karsen, H., Bilici, A., Bilgili, S.G. and Evirgen, O. (2008) Cutaneous Anthrax in Eastern Turkey: A Review of 85 Cases. Clinical and Experimental Dermatology, 33, 406-411. http://dx.doi.org/10.1111/j.1365-2230.2008.02742.x 
[17] Kanski, J.J. (2003) Clinical Ophthalmology, a Systematic Approach. 5th Edition, Butterworth Heinemann, Elsevier Science Limited, Edinburgh, 9.

[18] American Academy of Ophthalmology (2004) Basic and Clinical Science Course. Orbit, Eyelids, and Lacrimal System: Section 7, 2004-2005. American Academy of Ophthalmology, San Francisco, 44. 